\title{
LOCALIZATION OF GENERALIZED SPIKE AND WAVE DISCHARGE AND VALPROATE RESPONSE
}

Investigators at Universities of Cincinnati, $\mathrm{OH}$, and Birmingham, Alabama; and Montreal Neurological Institute, Canada, studied the EEG and functional magnetic resonance imaging (fMRI) in 89 patients with idiopathic generalized epilepsy (IGE), (25 with generalized spike and wave discharges (GSWD) identified in EEG), and compared patients with valproate (VPA)-refractory and VPA-responsive IGE. The fMRI blood oxygen-level dependent (BOLD) correlates of GSWD in the entire group of patients involved midline thalamus, frontal regions and temporal lobes. A comparison of VPAresponsive and VPA-resistant patients showed BOLD signal increases in the VPAresistant patients in medial frontal cortex, along the paracingulate gyrus and anterior insula bilaterally. VPA-resistant and VPA-responsive patients have different GSWD generators that may explain the reason for different responses and resistance to VPA in some cases. (Szaflarski JP, Kay B, Gotman J, Privitera MD, Holland SK. The relationship between the localization of the generalized spike and wave discharge generators and the response to valproate. Epilepsia 2013 Mar;54(3):471-80). (Response: Jerry P Szaflarski, Department of Neurology, UAB Epilepsy Center University of Alabama at Birmingham, AL 35294. E-mail: szaflaj@uab.edu).

COMMENT. The combining of EEG and fMRI is a noninvasive method of investigation of brain regions involved at the time of epileptic discharges (Gotman J, Pittau F. Epilepsia 2011 Jul;52 Suppl 4:38-42). Neuronal discharges during an interictal spike or spike-wave burst result in increased metabolism and blood flow, reflected in the blood oxygen-level dependent (BOLD) signal measured by fMRI. EEG-fMRI helps localize epileptic foci in nonlesional frontal lobe epilepsy; it also demonstrates thalamic involvement in generalized epileptic discharges.

\section{UPDATED ILAE REVIEW OF ANTIEPILEPTIC DRUG EFFICACY}

The International League Against Epilepsy (ILAE) Subcommission on AED Guidelines reviewed the literature from July 2005 to March 2012 and combined results with previous analysis (2006) to provide a comprehensive update of level of AED efficacy as initial monotherapy with newly diagnosed or untreated epilepsy. Class of study (I, II, and III) and level of efficacy (A, B, C, and D) are recorded. The combined analysis (1940-2012) includes a total of 64 randomized controlled trials (RCTs) [7 with class I evidence, 2 with class II], and 11 meta-analyses.

In children with partial-onset seizures, 2 RCTs were class III because of an openlabel design, too short treatment duration, and a forced exit criteria, and 4 new metaanalyses included OXC versus PHT, LTG versus CBZ, and CBZ versus OXC. CBZ is most frequently studied $(n=12)$ followed by VPA $(n=7)$ and PHT $(n=6)$. OXC is the only adequate comparator for childhood partial-onset seizures and is established (level A); CBZ, PB, PHT, TPM, VPA, and VGB are possibly (level C); and clobazam (CLB), CZP, LTG, and ZNS are potentially (level D) efficacious/effective as initial monotherapy.

In children with generalized-onset tonic-clonic seizures, CBZ, PB, PHT, TPM, and VPA are possibly (level C) and OXC is potentially (level D) efficacious/effective, 